\title{
Walnut inclusion in a palm-based high-fat and high-cholesterol diet without changing total energy supply stabilises advanced atheroma plaque through an anti-inflammatory mechanism in ApoE-deficient mice

\author{
Lázaro I ${ }^{1,2}$, Cofan $\mathrm{M}^{1,3}$, Surra J1,4, Gómez-Guerrero $\mathrm{C}^{5,6}$, Ortega $\mathrm{E}^{1,3}$, Osada J1,7, Dantas AP², Sala-Vila A $^{1,3}$
}

${ }^{1}$ CIBER de Fisiopatología de la Obesidad y Nutrición, ISCIII, Madrid; ${ }^{2}$ Experimental Cardiology, Institut Clínic Cardiovascular, Institut d'Investigacions Biomèdiques August Pi i Sunyer (IDIBAPS), Barcelona ; ${ }^{3}$ Lipid Clinic, Endocrinology and Nutrition Service, Hospital Clínic-IDIBAPS, Barcelona; ${ }^{4}$ Dept. Producción Animal, Escuela Politécnica Superior de Huesca, Huesca ; ${ }^{5}$ Renal, Vascular and Diabetes Research Lab, IIS-Fundación Jiménez Díaz,

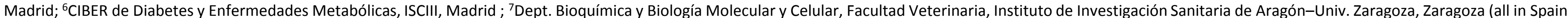
Contact e-mail: iolazaro@clinic.cat

\section{BACKGROUND AND AIM}

The North-to-South gradient in cardiovascular disease in Europe could be partially explained by the low prevalence of lipid-rich atheroma plaques (responsible for most culprit lesions) in Southern Europe. Consumption of certain foods delays atherosclerosis progression. In a mouse model of accelerated atherosclerosis we investigated whether the inclusion of walnuts within an atherogenic diet stabilizes advanced atheroma plaque

\section{RESULTS}

No differences in lesion extension and maximum lesion area
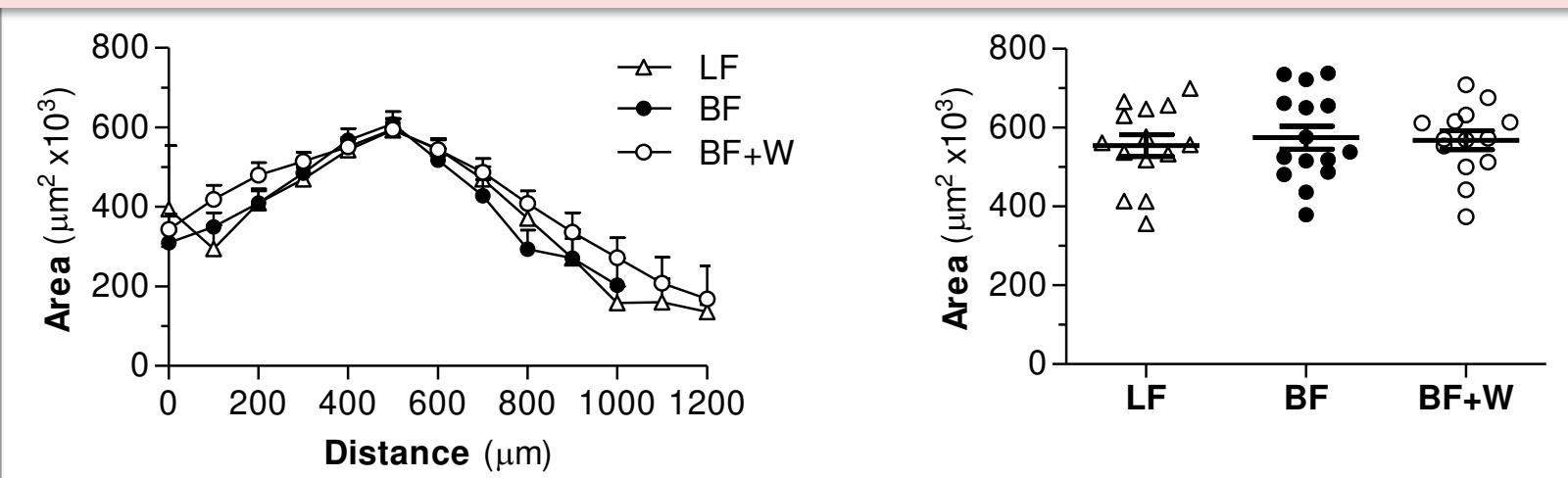

Inclusion of walnuts partially reverts features of unstable plaque
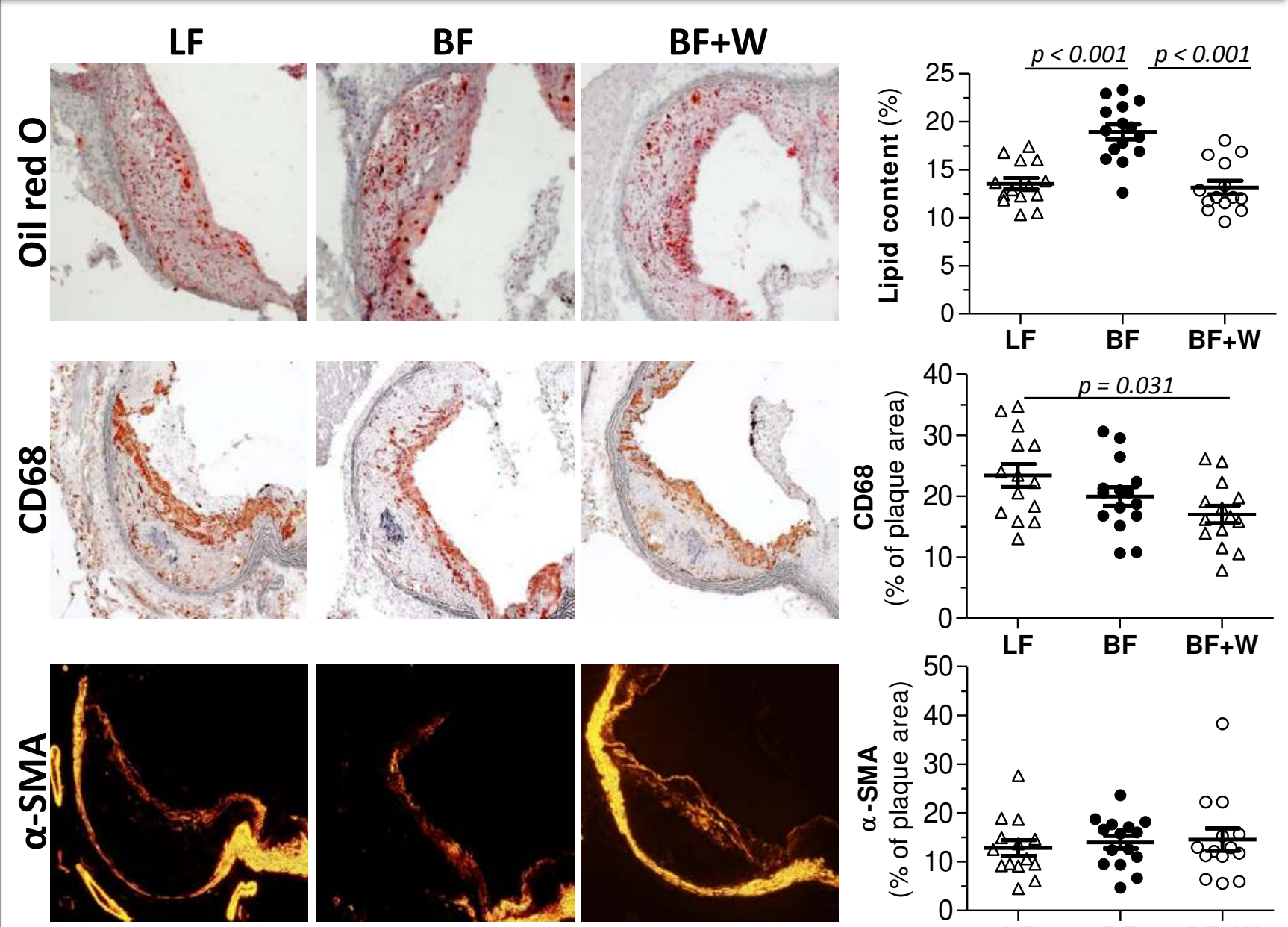

嶒
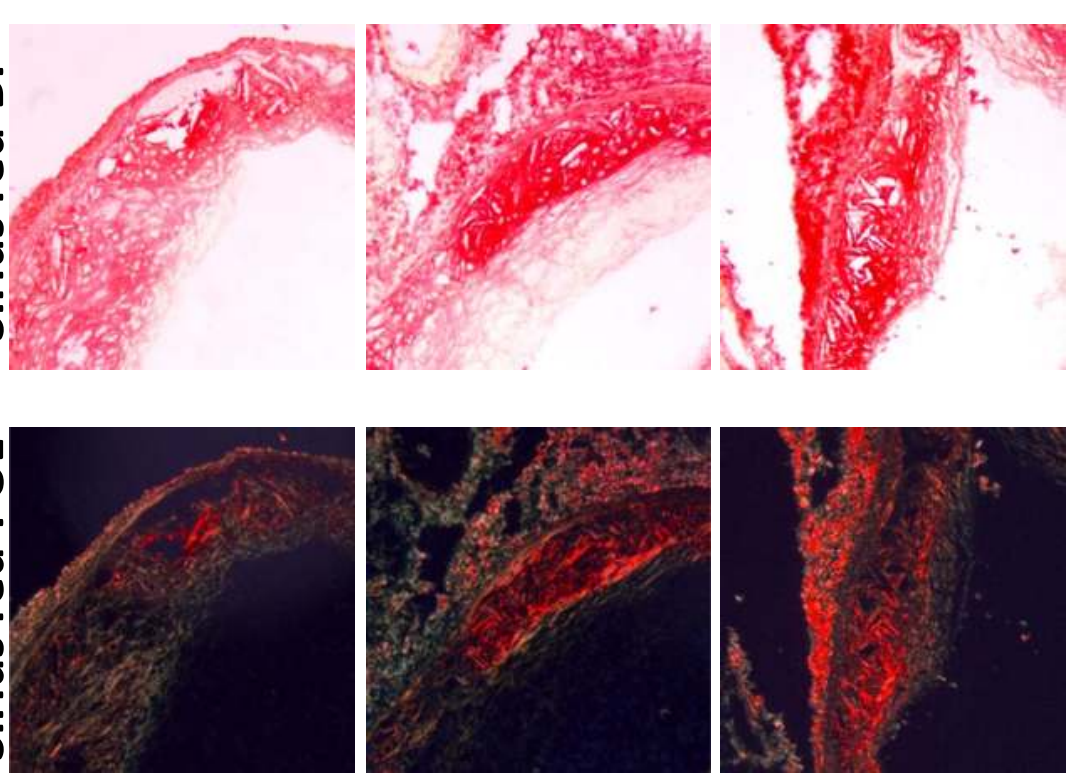

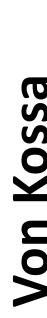
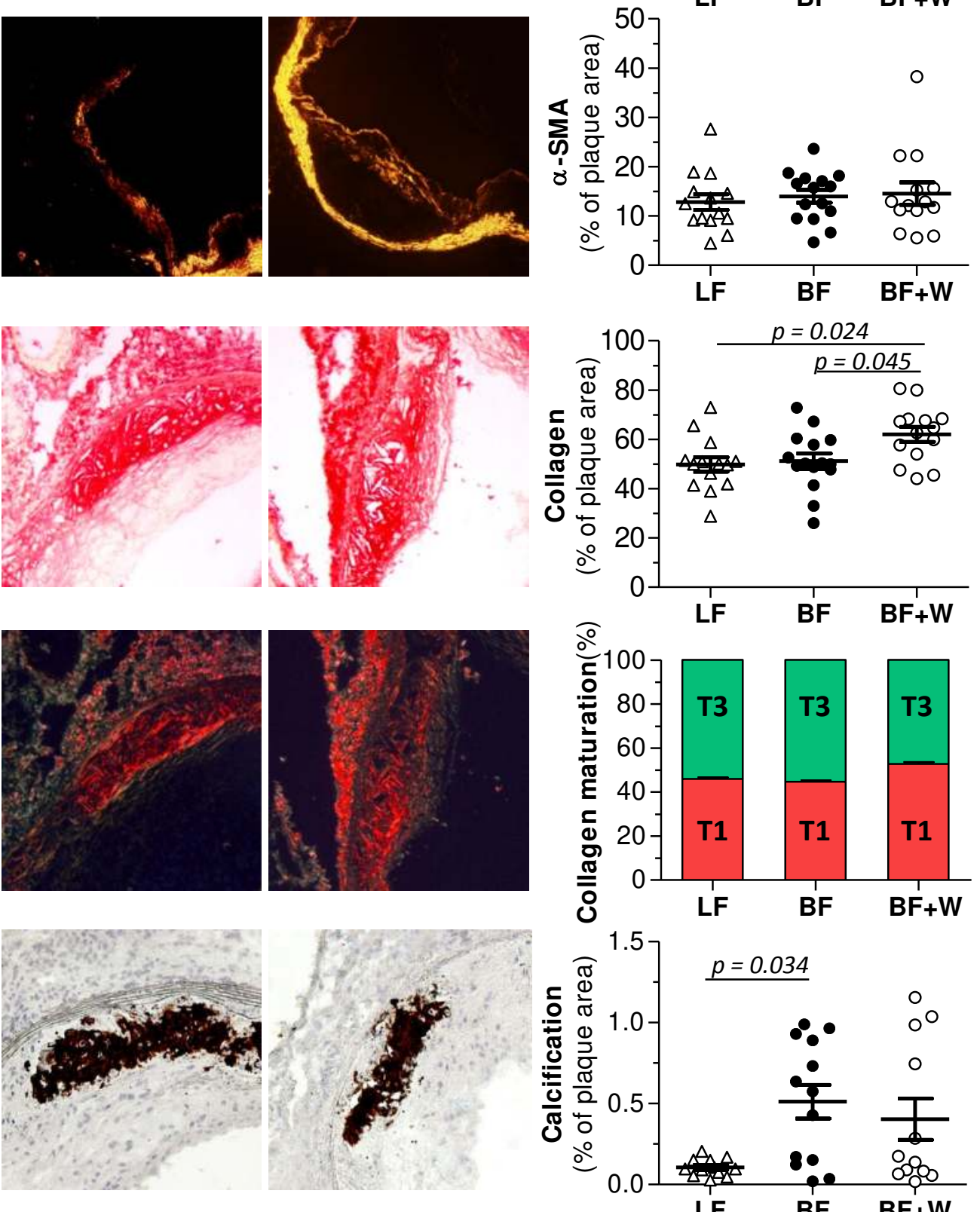

\section{Stary method:}

Grade I: foam cells

Grade II: foam cells, VSMC, few cholesterol clefts Grade III: foam cells, VSMC, numerous cholesterol clefts Grade IV: lipid core

\section{EXPERIMENTAL DESIGN}

\begin{tabular}{|c|c|c|c|c|c|}
\hline$\Gamma$ & $T$ & T & $T$ & $T$ & \\
\hline 10 & 13 & 16 & 19 & 22 & 25 age (wks) \\
\hline 0 & 3 & 6 & 9 & 12 & $15 \operatorname{diet}$ (wks) \\
\hline
\end{tabular}

SACRIFICE

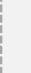

BAD FAT + WALNUT (BF+W; 0.2\% chol, isocaloric, palm oil $+3 \mathrm{~g}$ walnuts $/ 100 \mathrm{~g}, \mathrm{n}=14$

Walnut inclusion mitigates inflammatory gene expression

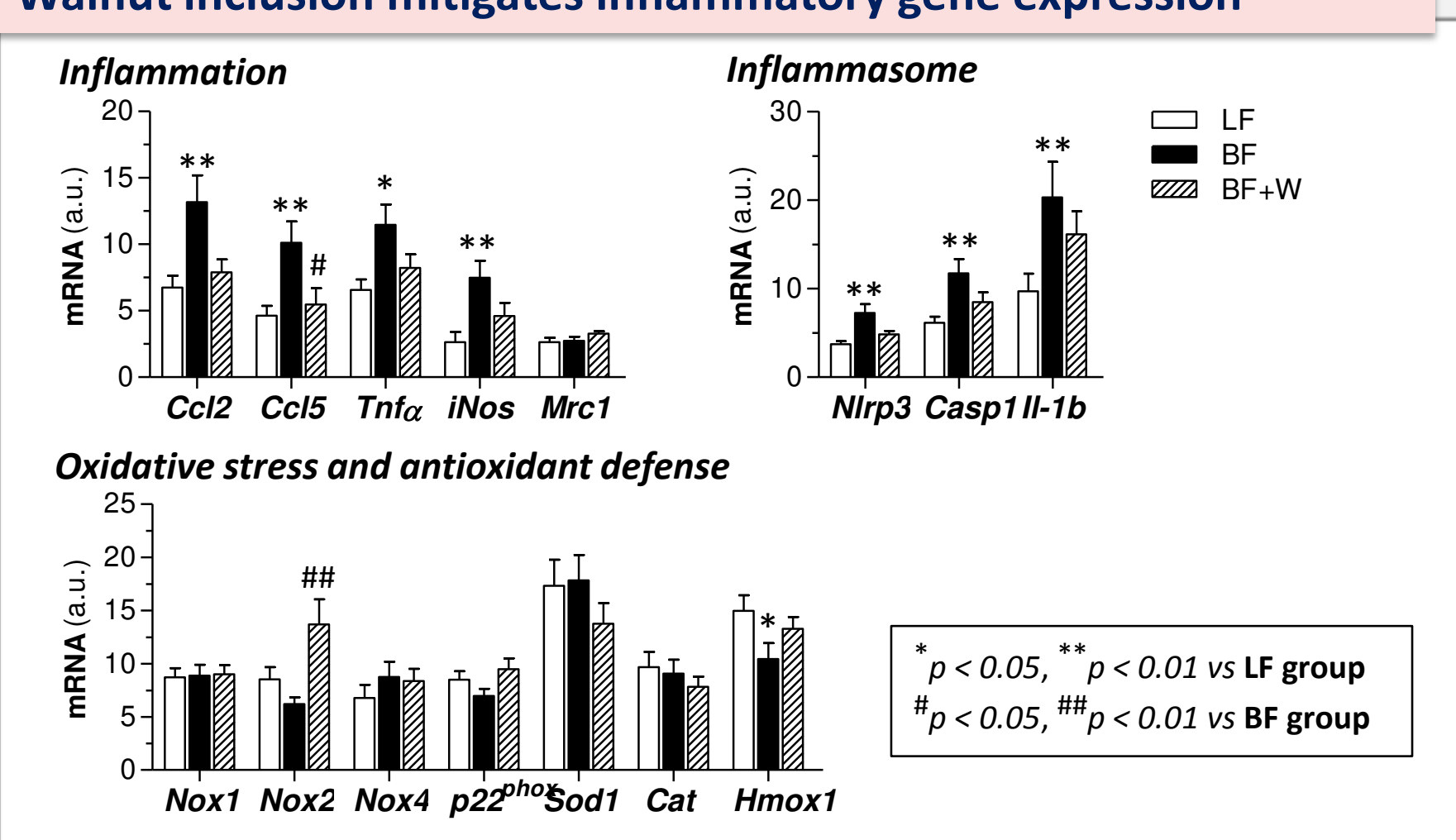

The effects of walnuts are likely explained by changes in NFKB and Nrf2 activation
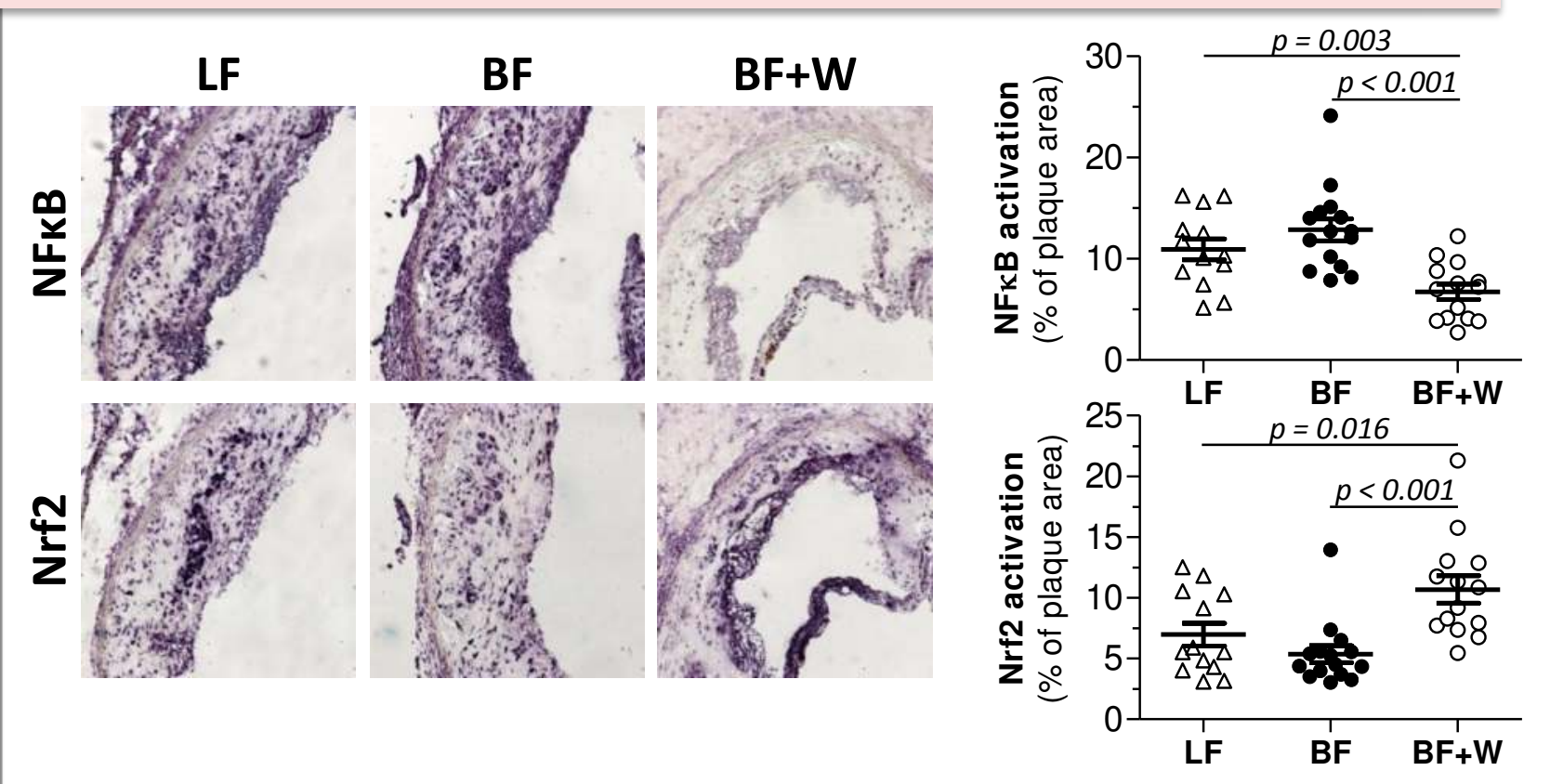

The effect of walnuts in plaque lipid content is beyond changes in circulant c-LDL

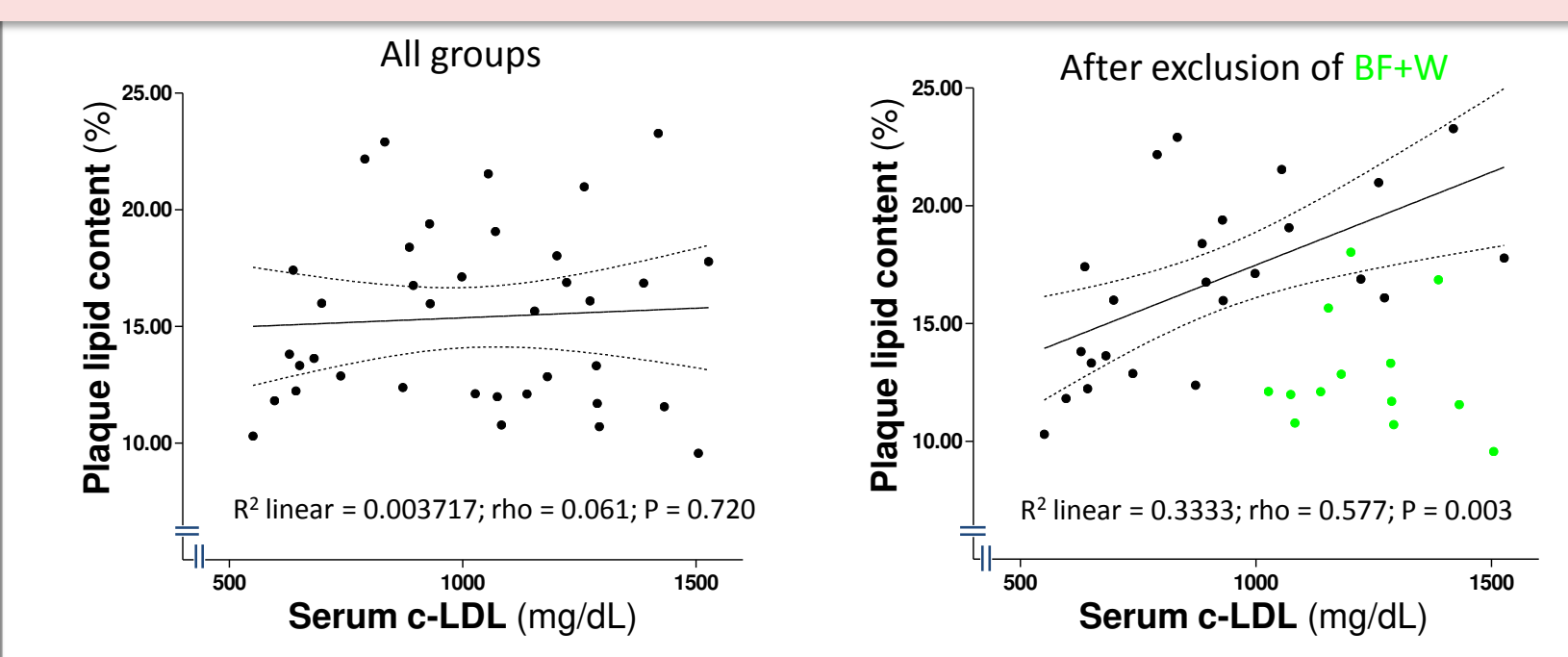

\section{CONCLUSION}

In an experimental model of atherosclerosis, isocaloric inclusion of walnuts in an unhealthy high-fat diet stabilizes advanced atheroma plaque. This contributes novel mechanistic evidence for the cardiovascular benefits of walnuts. 